\title{
AVALIAÇÃO DA INFLUÊNCIA DO PROCESSO DE SÍNTESE NA MASSA MOLAR, VISCOSIDADE, TAMANHO DE PARTÍCULA E DISTRIBUIÇÃO DE TAMANHO DE PARTÍCULA DE DISPERSÕES AQUOSAS DE POLIURETANO
}

\author{
V. M. S. de OLIVEIRA ${ }^{1}$, R. LIGABUE ${ }^{2}$, R. ROCHA ${ }^{2}$ \\ ${ }^{1}$ Universidade Federal Do Rio Grande do Sul, Programa de Pós-Graduação em Engenharia de Minas, \\ Metalúrgica e de Materiais \\ ${ }^{2}$ Pontifícia Universidade Católica do Rio Grande do Sul, Programa de Pós-Graduação em Engenharia \\ e Tecnologia de Materiais \\ E-mail para contato: vitmso@hotmail.com
}

\begin{abstract}
RESUMO - Dispersões aquosas de poliuretano (PUDs) têm sido utilizadas na área de revestimentos, adesivos e selantes como uma alternativa ecológica frente aos produtos base solvente por não emitirem compostos orgânicos voláteis. Na preparação desses sistemas, os parâmetros reacionais influenciam características como massa molar, viscosidade e tamanho de partícula desses sistemas. Este trabalho visa avaliar como o processo do pré-polímero e o processo da acetona influenciam nas propriedades das PUDs. Para tanto, foi realizada a síntese de PUDs pelos processos acima citados, onde os reagentes de partida foram o tetrametilxileno diisocianato (TMXDI) e um poliol poliéster linear, $\mathrm{MM}=1000 \mathrm{~g} / \mathrm{mol}$. As PUDs sintetizadas foram caracterizadas por espectroscopia vibracional no infravermelho (IV), cromatografia de permeação em gel (GPC), tamanho de partícula e viscosidade Brookfield. O espectro de infravermelho deste material apresentou uma banda intensa em torno de $1730 \mathrm{~cm}^{-1}$, duas bandas em torno de $1242 \mathrm{~cm}^{-1}$ e $1180 \mathrm{~cm}^{-1}$ características de grupo éster e uma banda de média intensidade em 1084 cm-1 característica de uretano (NH-COO). O filme polimérico das PUDs sintetizadas apresentou massa molar ponderal média em torno de $40000 \mathrm{~g} / \mathrm{mol}$. A viscosidade obtida das PUDs possui valores entre
\end{abstract} 175-19500 mPa.s, enquanto o tamanho de partícula médio está entre 110-227 nm.

\section{INTRODUÇÃO}

A contínua redução nos custos e controle da emissão de compostos orgânicos voláteis (VOCs, do inglês, volatile organic compounds) tem motivado o desenvolvimento de dispersões aquosas de poliuretano (PUDs, do inglês polyurethane dispersions) (Cakić et al., 2010, Garcia-Pacios et al., 2013 e Patel et al., 2010). Esses produtos apresentam muitas vantagens frente aos produtos convencionais base solvente, como por exemplo, a baixa viscosidade e boa aplicabilidade, segundo Cakić et al. (2010), podendo serem empregados em formulações de adesivos, revestimentos (Garcia-Pacios et al., 2013) e tintas base água (Fang et al., 2014), por exemplo. 


\section{9 a 22 de outubro de 2014 \\ Florianópolis/SC}

Segundo García-Pacios et al. (2013) PUDs consistem em uma cadeia de poliuretano termoplástico linear dispersa em água devido a presença de grupos iônicos em sua estrutura, que atuam como emulsificante interno. Normalmente, uma razão $\mathrm{NCO} / \mathrm{OH}$ maior que 1,5 é utilizada, resultando em um pré-polímero com grupos NCO terminais e posteriormente é realizada a extenção de cadeia com glicol formando grupos uretano. De acordo com Cakić et al. (2010) extensores de cadeia de baixa massa molar (MM) hidroxilados ou terminados em aminas fornecem importante função na morfologia de fibras poliuretanas, elastômeros, adesivos entre outros. As propriedades de PUDs termoplásticas são geralmente determinadas pela interação entre os segmentos rígidos e flexíveis e pela interação entre os grupos iônicos. Os segmentos flexíveis (i.e. cadeia do poliol) fornecem flexibilidade ao poliuretano, enquanto os segmentos rígidos (formado pela reação entre o isocianato e o extensor de cadeia) são duros e mais polares e possuem menor massa molecular (Garcia-Pacios et al., 2013).

Inúmeros estudos sobre esses sistemas têm sido publicado nos últimos anos, já que as propriedades de PUDs estão diretamente relacionadas aos parâmetros reacionais, tais como, tipo de poliol, tipo de isocianato, razão $\mathrm{NCO} / \mathrm{OH}$, teor de emulsificante interno e tipo de extensor de cadeia, porém não há ainda um estudo sobre as características de PUDs sintetizadas através de processos diferentes. Este trabalho visa caracterizar PUDs sintetizadas por dois processos usuais: o processo do pré-polímero e o processo da acetona.

\section{MATERIAIS E MÉTODOS}

As reações de síntese de dispersões aquosas de poliuretano (PUDs) foram realizadas em um reator de vidro, equipado com agitação mecânica, controle de temperatura, condensador de refluxo e entrada de gás inerte $\left(\mathrm{N}_{2}\right)$. Em todas as reações se utilizou excesso de diisocianato (razão equimolar $\mathrm{NCO} / \mathrm{OH}$ de 1,5 e 1,7), $5 \%$ de emulsificante interno, $90 \%$ de extensão de cadeia e $120 \%$ de neutralização. Todos os reagentes foram utilizados sem prévia purificação.

\subsection{Síntese de PUD pelo processo do pré-polímero}

Em uma reação típica, o reator foi carregado com um poliol poliéster linear (Degussa; $\mathrm{MM}=$ $1000 \mathrm{~g} / \mathrm{mol}$; funcionalidade = 2), o emulsificante interno, fornecedor de grupos iônicos, ácido dimetilol propiônico, DMPA (Rudnik; funcionalidade $=2$ ) e o catalisador dibutil dilaurato de estanho, DBTDL (Miracema-Nuodex Ind.) sob atmosfera inerte. Atingindo-se a temperatura desejada $\left(80{ }^{\circ} \mathrm{C}\right)$, foi adicionado lentamente o diisocianato meta-tetrametilxileno diisocianato, TMXDI (Cytec; pureza = 98,1 \%; funcionalidade =2), através de um funil de adição. Após, a temperatura foi elevada até 110 ${ }^{\circ} \mathrm{C}$ e procedeu-se a reação fazendo-se o controle do residual de NCO através da titulação com $n$ dibutilamina (Subramani, 2004 e Moss, 1997). A reação foi considerada completa quando o teor de NCO residual foi estabilizado. Em seguida, com a temperatura reduzida para valores abaixo de $40{ }^{\circ} \mathrm{C}$, adicionou-se $5-10 \%$ em massa de acetona para reduzir a viscosidade do meio e, após foi realizada a etapa de neutralização através da adição de trietilamina, TEA. Posteriormente, seguiu-se a etapa da dispersão e extensão de cadeia, que consiste em pesar, em um béquer de $1000 \mathrm{~mL}$, água deionizada e o extensor de cadeia etilenodiamina, EDA (Vetec; pureza $=98 \%$ ), e a essa solução, sob agitação vigorosa $(800-1500 \mathrm{rpm})$, com um funil de adição foi adicionado o pré-polímero, à temperatura próxima da ambiente. 


\subsection{Síntese de PUD pelo processo da acetona}

Os principais reagentes, TMXDI, poliol, catalisador e DMPA, foram colocados no reator e a temperatura foi elevada a $105{ }^{\circ} \mathrm{C}$. Alíquotas foram retiradas para verificação do teor de NCO residual pelo método da $n$-dibutilamina e quando esse se estabilizou, a temperatura foi reduzida e adicionou-se aproximadamente 30 - $40 \%$ (em massa) de acetona. Seguiu-se com a neutralização com TEA e, posteriormente, foi adicionada a solução aquosa de extensor de cadeia à solução de pré-polímero em acetona.

\subsection{Retirada dos solventes orgânicos}

Após a dispersão, os solventes orgânicos foram retirados com o auxílio de um rotovapor ou com uma linha de vácuo até a obtenção do teor de sólidos calculado para a quantidade de água adicionada (teor de sólidos de $~ 40 \%$ ).

\subsection{Técnicas de caracterização}

As PUD sintetizadas foram caracterizadas por análise de teor de sólidos, ou não voláteis (NV), medidas de $\mathrm{pH}$, viscosidade Brookfield, tamanho de partícula médio e distribuição de tamanho de partícula. Os filmes das PUDs sintetizadas foram solubilizados em tetraidrofurano (THF) e caracterizados por espectroscopia vibracional na região do infravermelho (IV) e cromatografia de permeação em gel (GPC). Esses fimes foram preparados em uma placa de vidro, aplicados com um extensiômetro de $100 \mu \mathrm{m}$ e secos ao ar por uma semana.

\section{RESULTADOS E DISCUSSÃO}

Foram realizadas quatro reações de síntese de PUDs, onde se variou o processo e a razão $\mathrm{NCO} / \mathrm{OH}$, esses dados estão sendo mostrados na Tabela 1.

Tabela 1 - Reações de síntese de PUDs e suas variações

\begin{tabular}{c|c|c}
\hline Dispersões & Processo & NCO/OH \\
\hline PUD 1 & Pré-polímero & 1,7 \\
PUD 2 & Pré-polímero & 1,5 \\
PUD 3 & Acetona & 1,7 \\
PUD 4 & Acetona & 1,5 \\
\hline
\end{tabular}

As PUDs 1 e 2 foram sintetizadas pelo processo do pré-polímero, enquanto que para as PUDs 3 e 4 utilizou-se o processo da acetona. Para comparação, PUDs sintetizadas pelo mesmo processo tiveram suas razões $\mathrm{NCO} / \mathrm{OH}$ variadas: PUDs 1 e 3 com razão NCO/OH = 1,7 e PUDs 2 e 4, com razão $\mathrm{NCO} / \mathrm{OH}=1,5$. 
As PUDs sintetizadas foram caracterizadas através da técnica de espectroscopia vibracional na região do infravermelho (IV), sendo as atribuições das bandas realizadas em comparação aos valores das freqüências características para os grupos existentes na molécula, de acordo com a literatura (Silverstein, 1994). A Figura 1 apresenta o espectro de IV típico de filmes de PUDs sintetizadas com o poliol poliéster linear e TMXDI.

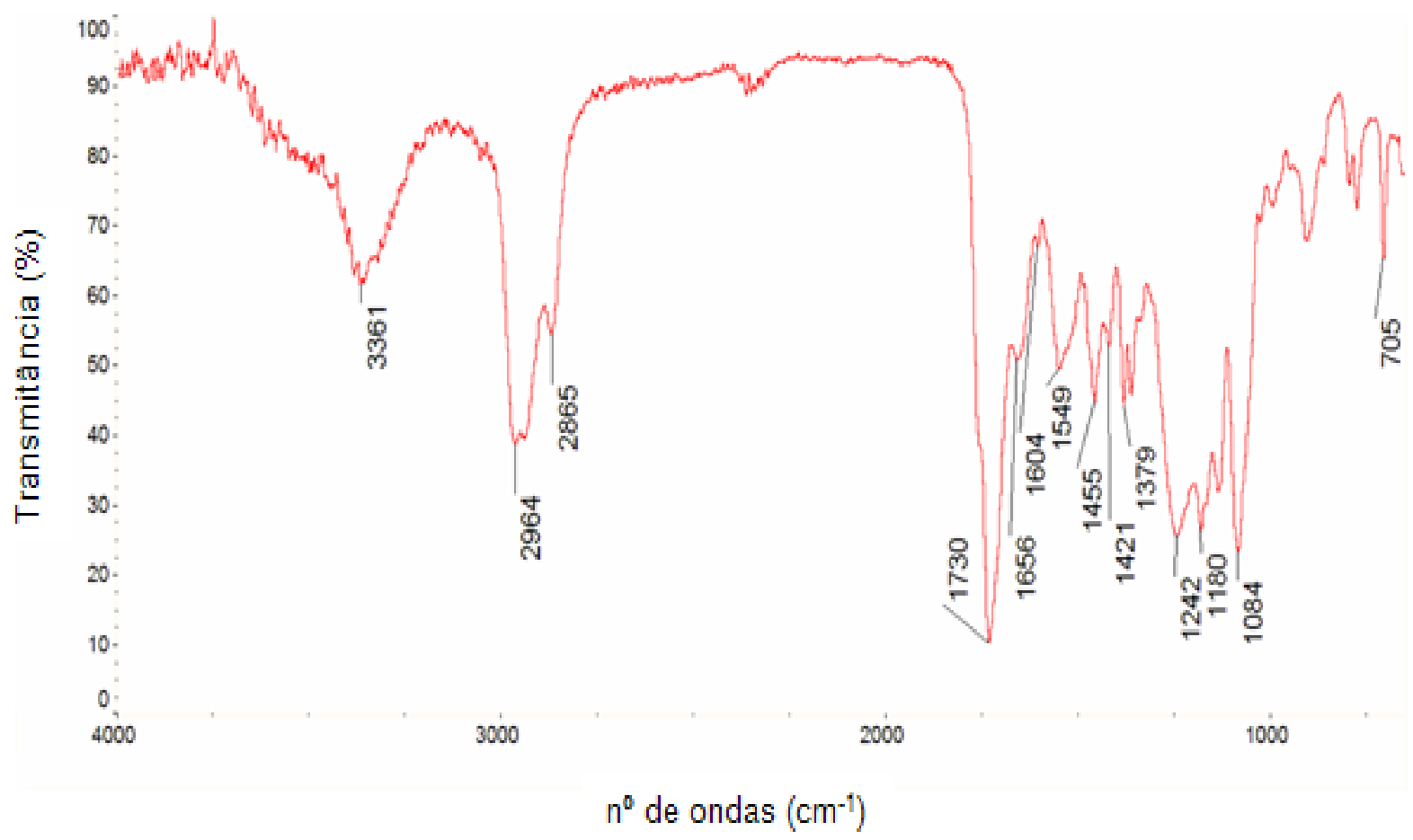

Figura 1 - Espectro de IV típico de filme de PUD sintetizada com poliol poliéster linear e TMXDI.

Por motivo de falta de espaço só serão apresentadas aqui, as atribuições mais importantes das bandas apresentadas neste espectro. O espectro de IV, mostrado na Figura 1, apresenta banda característica da ligação $\mathrm{N}-\mathrm{H}$ de uretano em $3361 \mathrm{~cm}^{-1}$. A banda característica do grupo NCO livre residual em torno de $2115 \mathrm{~cm}^{-1}$ não é encontrada, confirmando a reação total entre os grupos NCO do diisocianato com o poliol e com o extensor de cadeia, o que é desejado, já que NCO livre é tóxico e a existência de NCO livre pode gerar reações secundárias indesejáveis (por exemplo, com a água gerando ácido carbâmico que se decompõe para dar como produto final uréia) de acordo com Chattopadhyay e Raju (2007). Um alargamento na banda atribuída ao estiramento $\mathrm{C}=\mathrm{O}$ (uretano) $\left(1730 \mathrm{~cm}^{-1}\right)$ é observado no espectro apresentado, em função da sobreposição com a banda de estiramento $\mathrm{C}=\mathrm{O}$ de grupo éster. Na região de $1084 \mathrm{~cm}^{-1}$ tem-se uma banda de média intensidade característica do grupo N-CO-O de uretano (Otts e Marek, 2005). sintetizadas.

Na Tabela 2 são apresentados os resultados das demais análises realizadas para as PUDs 
Tabela 2 - Resultados das análises das PUDs sintetizadas

\begin{tabular}{c|c|c|c|c|c|c|c}
\hline Dispersões & Mn (g/mol) & Mw (g/mol) & I. P. & $\begin{array}{c}\text { NV } \\
(\%)\end{array}$ & pH & $\begin{array}{c}\text { T. P. } \\
(\mathrm{nm})\end{array}$ & $\begin{array}{c}\text { Viscosidade } \\
(\mathrm{mPa} . \mathrm{s})\end{array}$ \\
\hline PUD 1 & 23065 & 44228 & 1,92 & 45 & 8 & 227 & 19500 \\
PUD 2 & 24297 & 47918 & 1,97 & 45 & - & 184 & - \\
PUD 3 & 19843 & 36535 & 1,84 & 36 & 9 & 110 & 175 \\
PUD 4 & 28780 & 53070 & 1,84 & 41 & 9 & 121 & 10800 \\
\hline
\end{tabular}

Mn: massa molar numérica média; Mw: massa molar ponderal média; I. P.: índice de polidispersão; NV: não voláteis (teor de sólidos); T. P.: tamanho de partícula.

A partir dos resultados das análises de GPC, percebe-se que não houve grande influência do método de síntese (processo) nas massas molares dos polímeros obtidos. Pode-se dizer que no processo da acetona, uma razão $\mathrm{NCO} / \mathrm{OH}$ menor leva a um aumento na massa molar, mas essa variação não é significativa. O índice de polidispersão, I. P., é bastante próximo em todas as PUDs sintetizadas, o que significa que tanto o processo quanto a razão $\mathrm{NCO} / \mathrm{OH}$ não alteram significativamente a relação $M w$ e $M n$.

No processo da acetona é necessária a utilização de quantidade maior de acetona na etapa da dispersão para que a água possa solubilizar o pré-polímero, já que é feita a adição de água no prépolímero, ao contrário do processo do pré-polímero, em que este é adicionado à água. Provavelmente, por essa razão (maior quantidade de acetona) que se obteve $36 \%$ e $41 \%$ de não voláteis para as PUDs 4 e 5, respectivamente. Isso pode ser devido à maior quantidade de acetona adicionada nessas PUDs, pois esse solvente deve facilitar a saída de água.

Os valores de $\mathrm{pH}$ encontrados para as PUDs sintetizadas ficaram em torno de 8 e 9, levemente alcalinos, o que é justificado pela adição de excesso de amina, uma base, adicionada na etapa de neutralização $(120 \%)$.

A diferença no valor de viscosidade entre as dispersões mostrada na Tabela 2, é devida, provavelmente, ao alto valor de teor de sólidos obtidos nas dispersões PUD 1, 2 e 4, já que uma maior quantidade de segmento flexível (razão $\mathrm{NCO} / \mathrm{OH}=1,5$ na $\mathrm{PUD}$ 4) parece não afetar significativamente os valores de massa molar do polímero poliuretano (PU) disperso. A PUD 2, sintetizada pelo processo do pré-polímero, apresentou alta viscosidade, se tornou uma pasta e a viscosidade e o pH não puderam ser medidos. Essa elevação abrupta na viscosidade pode ser relacionada com o menor tamanho de partícula quando comparada com a PUD 1, já que quanto menor a partícula, maior a viscosidade, segundo dados da literatura, em Nanda e Wicks (2006) e Yen (2006).

Ao analisar os valores de tamanho de partícula médio (Tabela 2) pode-se concluir que o processo da acetona fornece produtos com tamanho de partícula médio menor do que aquelas produzidas pelo processo do pré-polímero. Conforme Fang et al. (2014), menor tamanho de partícula médio é preferível para que se obtenha boa penetração no substrato. 
Os valores de tamanho de partícula apresentados são valores médios. Uma informação também importante é a distribuição do tamanho de partícula, que pode ser visto na Figura 2, que apresenta os gráficos de distribuição de tamanho de partícula médio das PUDs sintetizadas.
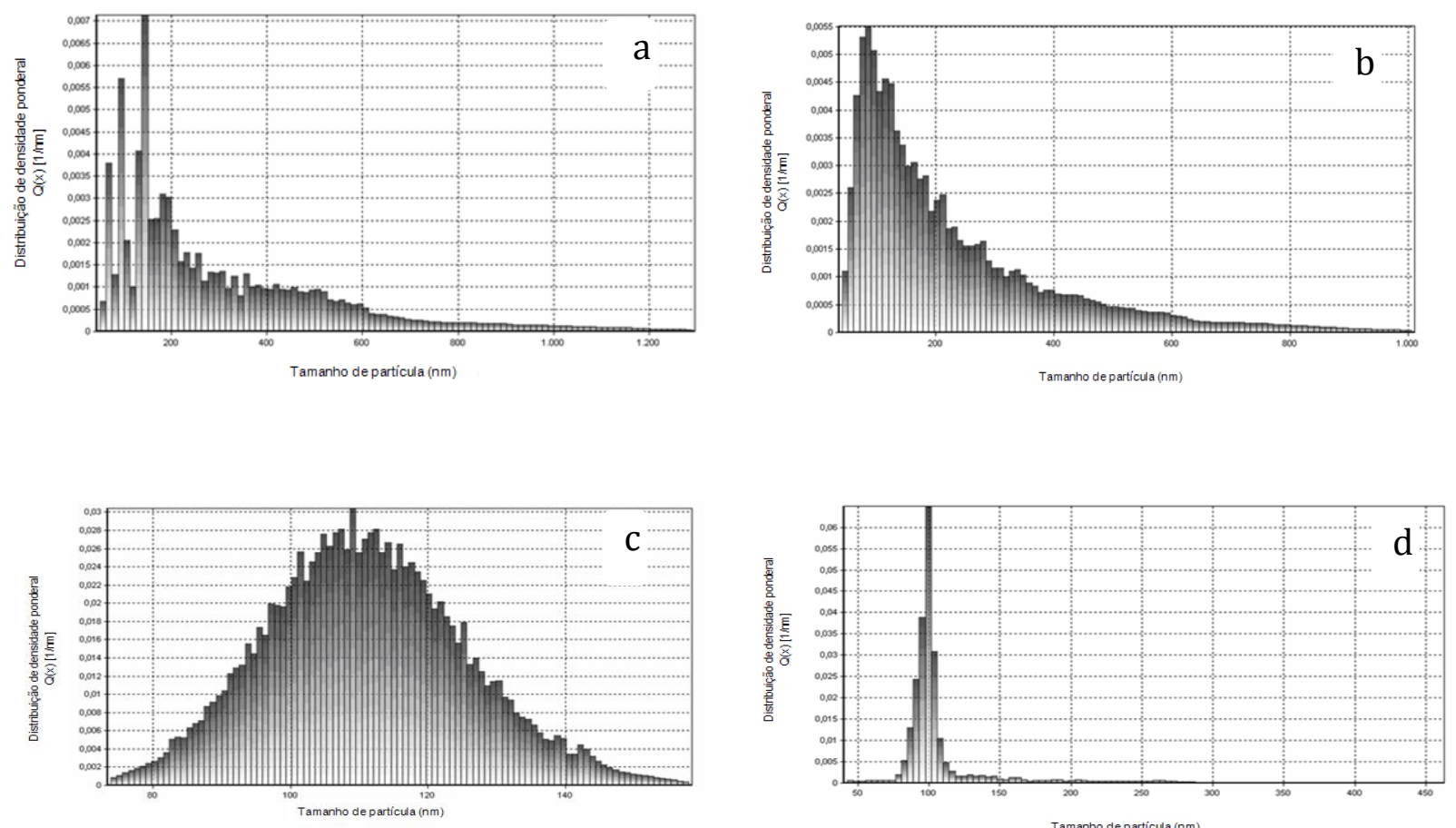

Figura 2 - Gráficos de distribuição de tamanho de partícula: (a) PUD 1, (b) PUD 2, (c) PUD 3 e (d) PUD 4.

Para as PUDs sintetizadas pelo processo do pré-polímero, PUD 1 e 2 (Figura 2a e 2b, respectivamente), é notável a ampla e heterogênea distribuição de tamanho de partícula médio. A grande maioria das partículas dessas PUDs possui tamanho menor que $200 \mathrm{~nm}$, porém existem várias partículas com tamanho maior que $500 \mathrm{~nm}$, o que influencia significativamente na viscosidade da dispersão produzida. Este fato é confirmado por Pérez-Limiñana (2005) que, eu seu trabalho, também apresentou uma maior heterogeneidade na distribuição de partículas em termos de volume, e supôs que essa maior dispersão pode ser devido a excessiva viscosidade do pré-polímero durante a etapa de dispersão na produção da partícula iônica, produzindo partículas com formas mais alongadas, as quais são responsáveis pelas diferentes medidas de tamanho de partícula.

Analisando os gráficos de distribuição de tamanho de partícula, pode-se concluir, finalmente, que o processo da acetona (Figuras $2 \mathrm{~b}$ e $2 \mathrm{~d}$ ) produz partículas mais homogêneas, em relação ao tamanho, ou seja, sua distribuição ou polidispersão é menor que aquelas formadas pelo processo do pré-polímero (Figuras 2a e 2c), onde é possível notar uma ampla e heterogênea distribuição de tamanho de partícula. Essas diferenças na distribuição de tamanho de partícula médio entre as reações são, provavelmente, devidas à etapa da dispersão, pois é nesta etapa que as micelas são formadas e dependendo da velocidade de rotação, da viscosidade do pré-polímero, do tipo de hélice utilizada na 


\section{9 a 22 de outubro de 2014 \\ Florianópolis/SC}

dispersão entre outros fatores, as micelas podem apresentar tamanhos diferentes. O processo da acetona moutrou-se ser o mais adequado quando há a necessidade de homogeneidade no tamanho das partículas de PUDs.

\section{CONCLUSÃO}

Através do estudo realizado pode-se verificar a influência do método de síntese, ou processo, no tamanho de partícula, na distribuição do tamanho de partícula e na viscosidade de dispersões aquosas de poliuretano. Para diferentes tipos de aplicação desse material deve-se recorrer a um processo ou outro. Verificou-se que no processo da acetona, teores de sólidos mais baixos são obtidos, diminuindo a viscosidade do produto final. Em contrapartida, através desse processo se obtém partículas menores, o que leva a aumento na viscosidade, segundo Li et al. (2007). Conclui-se então que o processo da acetona produz dispersões com tamanho de partícula médio menor e com distribuição de tamanho de partícula mais homogêneo.

\section{REFERÊNCIAS}

CAKIĆ, S. M.; RISTIĆ, I. S.; DJORDJEVIĆ, D. M.; STAMENKOVIĆ, J. V.; STOJILJKOVIĆ, D. T. Effect of the chain extender and selective catalyst on thermooxidative stability of aqueous polyurethane dispersions. Progress in Organic Coating, v. 67, p. 274-280, 2010.

CHATTOPADHYAY, D. K.; RAJU, K. V. S. N. Structural engineering of polyurethane coatings for high performance applications. Progress in Polymer Science, v. 32, p. 352-418, 2007.

COUTINHO, F.; DELPECH, M. Síntese e caracterização de poliuretanos em dispersão aquosa à base de polibutadieno líquido hidroxilado e diferentes diisocianatos. Polímeros: ciência e tecnologia, v. 12, n. 4, p. 248-254, 2002.

FANG, C.; ZHOU, X.; YU, Q.; LIU, S.; GUO, D.; YU, R.; HU, J. Synthesis and characterization of low crystalline waterborne polyurethane for potential application in water-based ink binder. Progress in Organic Coatings, v. 77, p. 61-71, 2014.

GARCÍA-PACIOS, V.; JOFRE-RECHE，J. A.; COSTA，V.; COLERA， M.; MARTÍNMARTÍNEZ, J. M. Coatings prepared from waterborne polyurethane dispersions obtained with polycarbonates of 1,6-hexanediol of different molecular weights. Progress in Organic Coatings, v. 76, p.1484-1493, 2013.

LI, Q. N.; SUN, D. C. Synthesis and characterization of high solid contend aqueous polyurethane dispersion. Journal of applied polymer science, v. 105, p. 2516-2524, 2007.

MOSS, Michelle. Polyurethane dispersions for adhesive applications. Pigment \& Resin 
Technology, v. 26, n. 5, p. 296-299, 1997.

NANDA, A.; WICKS, D. The influence of the ionic concentration, concentration of the polymer, degree of neutralization and chain extension on aqueous polyurethane dispersions prepared by the acetone process. Polymer, v. 47, p. 1805-1811, 2006.

OTTS, D.; URBAN, M. Heterogeneous crosslinking of waterbourne two-component polyuretanes (WB 2K-PUR); stratification processes and the role of water. Polymer, v. 46, p. 2699-2709, 2005.

PATEl, A.; PATEL, C.; PATEL, M.G.; PATEL, M.; DIGHE, A. Fatty acid modified poluyretane dispersion for surface coatings: Effect os fatty acid contend and ionic contend. Progress in Organic Coatings, v. 67, p. 255-263, 2010.

PÉREZ-LIMIÑANA, M. A.; ARÁN-AÍS, F.; TORRÓ-PALAU, A. M.; ORGILÉS-BARCELÓ, A. C.; MARTÍN-MARTÍNEZ, J. M. Characterization of waterborne polyurethane adhesives containing different amounts of ionic groups. International Journal oF Adhesion \& Adhesives, v. 25, p. 507-517, 2005.

SILVERSTEIN, R. M.; BASSLER, G. C.; MORRIL, T. C. Identificação Espectrométrica de Compostos Orgânicos. Rio de Janeiro: Guadanabara, 1994.

SUBRAMANI, S.; CHEONG, I. W.; KIM, J. H. Synthesis and characterizations of silylated polyurethane from methyl ethyl ketoxime-blocked polyurethane dispersions. European Polymer Journal, v. 40, p. 2745-2755, 2004.

YEN, M. S; TSAI, P. Y.; HONG, P. D. The solution properties and membrane properties of polydimethylsiloxane waterborne polyurethane blended with the waterborne polyurethanes of various kinds of soft segments. Colloids and Surfaces A: Physichem. Eng. Aspects, v. 279, p. 1-9, 2006. 